\title{
What the First Issue of 2017 BRN Reviews Features...
}

It is acknowledged that chronic obstructive pulmonary disease (COPD) represents a very serious overall challenge to the global respiratory community. One of the best examples of such a statement is the article written by María Montes de Oca and Victorina López, former Asociación Latinoamericana del Tórax (ALAT) Presidents, in which the essential features of the PLATINO (Proyecto Latino-Americano de Investigación en Obstrucción Pulmonar) study are extensively reported. The PLATINO project is an extraordinary survey entirely developed in Latin America over the last 15 years to assess the burden of COPD in five low- and middle-income representative cities of this part of the world (São Paulo, Brazil, Santiago, Chile, Mexico City, Mexico, Montevideo, Uruguay, and Caracas, Venezuela). PLATINO, a brilliant initiative of ALAT, has filled in an important gap in the epidemiological knowledge of COPD in Latin America, along with the improvement of our current understanding of the main features of the disease from the study of a general population. Key aspects of the study include prevalence, diagnosis accuracy, and management and also risk factors, clinical patterns, follow-up, and diagnosis stability over time related to COPD in South America.

Aging is a progressive loss of physiological integrity, leading to impaired function and increased vulnerability to death. Many of the hallmarks that characterize the aging process are observed in COPD and idiopathic pulmenary fibrosis (IPF). Aging represents, nowadays, an emerging area in our understanding of these two chronic respiratory disorders, very elegantly co-authored by Mauricio Rojas and Jacob Sellarés from the University of Pittsburg $\overline{\hat{h}}$, the senior author with a renowned experience in the field. Accelerated aging of the lungs has been considered a relevant mechanism in these two chronic respiratory diseases. Thus, telomere attrition, loss of proteostasis, mitêchondrial dysfunction, cellular senescencề, and stem cell exhaustion have all been considered key mechanisms of aging, present in both clinical conditions. In this review, the biology of aging and its relationship with the pathogenesis of COPD and COPD are comprehensively addressed.

The third article introduces a remarkable respiratory researcher, José M. Marín, witha strong research background in the field of COPD and obstructive sleep apnoea (OSA). His review focuses on the coexistence of these two important chronic respiratory conditions that affect topographically upper airway and both large and small airways. In the begingning, the author rightly points out that the co-occurrence of COPD and OSA was originally named "overlap syndrome" by David Flenley about 30 years ago; today this is not officially considered a formal diagnostic term. 
The original proposal was to underline the use of polysomnography in patients with COPD with coexisting obesity, snoring, or morning headache associated with nocturnal oxygen therapy, to unravel the sleep-apnoea comorbidity in these patients. The presence of such clinical overlap, when OSA is untreated in patients with COPD, carries a higher risk of more adverse diurnal and nocturnal physiological and clinical events, including greater sleep fragmentation, more severe night-time gas exchange disturbances, and increased overall mortality than is reported for COPD and OSA each alone.

The fourth article, by Maria R. Bonsignore, Oreste Marrone and Walter T. McNicholas, a former European Respiratory Society (ERS) President, addresses the complex debate of the evidences for the therapeutic use of continuous positive airway pressure (CPAP) in OSA. This article provides updated evidence-based information on the effects of CPAP on mortality, cardio-metabolic outcomes, inflammatory biomarkers, sleepiness, car traffic casualties, cognitive dysfunction, and health-related quality of life. While the evidence to support the beneficial effects of CPAP treatment on mortality, systemic hypertension, daytime sleepiness and car accidents, and quality of life is solid, the evidence for cognitive dysfunction, cardiovascular outcomes, and metabolic and inflammatory markers still remains uncertain and subject to further studies.

The last review of the current issue is written by Joaquim Gea, Cristina Estirado and Esthềr Barreiro, a trio of first-class researchers with a long-time, prestigious experience in the fielâ. It is devoted to nutritional abnormalities in patients with COPD, one of the most prominent non-pulmonary features in this disease. This review comprehensively complements the prêvious one by J.M. Marín, addressing the coexistence of COPD and OSA. Nutritional diso ders are frequently observed in COPD patients and are diagnosed using anthropometry and determination of different body components. Low body weight and muscle mass result in muscle dysfunction, impaired exercise capacity, and worse outcomes, including increased mortality. The review ends with some practical recommendations for clinicians regarding the management and treatment of such nutritional abnormalities in the COPD field.

R. Rodriguez-Roisin, ME December 2016 